\title{
Modeling the hygrothermal response of a prefabricated straw bale panel and assessing its impact on indoor climate
}

\author{
S. Dubois $^{1 *}$, A. Evrard ${ }^{2}$, F.Lebeau ${ }^{1}$ \\ ${ }^{1}$ University of Liege, Gembloux Agro-Bio Tech, Passage des Déportés 2, 5030 Gembloux, Belgium \\ ${ }^{2}$ Catholic University of Louvain, Architecture et Climat, Place du Levant 1, 1348 Louvain-La-Neuve, Belgium \\ *Author supported by a FRIA Grant (FNRS, Belgium)
}

2014 ASABE and CSBE/SCGAB Annual International Meeting, Montreal, Quebec Canada, July 13 - 16, 2014

\begin{abstract}
Crop-based materials show many qualities in the quest for construction products with low environmental impact in the construction sector. Their ability to exchange moisture with indoor air offers new opportunities for improving interior user comfort and eventually buildings energy performance. Modeling their hygrothermal behavior has thus focused much attention, one of the main objectives being to assess more objectively the resulting performance at building scale. The first natural step in this modeling process is to understand the specific dynamics of heat and mass transfer within such materials. The Building Element Heat Air and Moisture (BEHAM) numerical models were designed to accurately predict internal conditions encountered in materials with partial balance equations. This envelope-based approach, however, requires the knowing of the interior and exterior climate conditions prior to the study. Therefore, it forbids any assessment of mutual exchange between the envelope to the indoor air and prevents any serious validation of the overall performance of materials. In response to this problem, the importance of developing whole building hygrothermal model has recently been raised. General scientific computational tools like MATLAB seem particularly adapted to perform this scaling-up, given their high flexibility and tools integration potentialities.

In this communication we study a prefabricated straw bale panel subjected to two distinct and simple solicitations in a climatic chamber. A whole building hygrothermal model developed in MATLAB is then validated with the experimental datasets. The evolution of indoor conditions under the different tests is analyzed in details with an assessment of straw bales performance.
\end{abstract}

Keywords. Building materials, Straw bales, Modeling, Heat flow, Energy balance, Moisture.

\section{Introduction}

The first step in assessing crop-based materials (CBM) performance is to understand the specific dynamics of heat and mass transfer occurring within them. Building Element Heat, Air and Moisture (BEHAM) numerical are designed to predict the internal conditions encountered in materials based on partial balance equations (PDE). However, those approaches based on individual materials or envelope parts require to know the interior and exterior climatic conditions which are imposed to the studied element. Therefore it prevents any detailed assessment of the impact of chosen materials on the indoor environment and therefore prohibits any concrete conclusion of the overall envelope performance in the context of an operating building.

In response to this problematic, the development of whole-building hygrothermal models has recently focused much attention in Building Physics research (Hens, 2005; Rode \& Grau, 2008; van Schijndel, 2009; Woloszyn \& Rode, 2008). Such models are obtained by combining a description of hygrothermal transfers in indoor air volumes with a description of the hygrothermal response of the envelope. By integrating these two types of descriptions, it becomes possible to characterize the evolution of the indoor climate of a zone or a building while considering the actual participation of envelope materials.

In 2005, the Annex 41 of the international Energy Agency (IEA) was a cooperative project on the whole-building heat, air and moisture response, part of the Energy Conservation in Buildings and Community Systems program (ECBCS). Seventeen tools were then already identified to perform some common modeling exercises where the indoor air of a room had to interact dynamically with the heat, 
air and moisture response of the envelope (Rode \& Woloszyn, 2007). In fact, those simulation tools differ in the level of spatial discretization they offer for the description of indoor air and envelope domains, sometimes referred to as the 'granularity'. (Janssens, Woloszyn, Rode, Sasic-Kalagasidis, \& De Paepe, 2008). The highest granularity for indoor air volume is the Computational Fluid Dynamics (CFD) modeling whereas the coarser consists of a perfectly mixed air assumption for the whole building. For envelope modeling, very fine models can provide a 3D description of coupled heat, air and moisture transfers in construction materials assembly. The coarser level uses simple model to characterize the envelope response, like lumped approaches, which doesn't provide an actual computation of temperature and moisture fields in the whole depth of porous walls (Janssens et al., 2008). Various combination of granularity for envelope and indoor air modeling are observed among models capable of whole-building hygrothermal modeling, often depending on their origin. Indeed, some originate from Building Energy Simulation models, and are generally characterized by a coarser granularity for envelope modeling, while others originate from BEHAM tools, and the air volume modeling is often really simplified. An extensive review of available tools can be found in (Woloszyn \& Rode, 2008).

Recently, some authors showed that general scientific computational tools like Matlab (Sasic Kalagasidis, 2004; Tariku, Kumaran, \& Fazio, 2010; van Schijndel, 2009) or SPARK (Tran Le, Maalouf, Mai, Wurtz, \& Collet, 2010) can be used to develop whole building hygrothermal models that meet important requirements for research oriented modeling. However, there are still very few validation studies in real full-scale environments (Salonvaara, Ojanen, Holm, Künzel, \& Karagiozis, 2004).

In this paper, a room-scale hygrothermal model is constructed by combining a research BEHAM model developed in COMSOL Multiphysics with a description of heat and moisture balance of the interior air. The BEHAM model can be translated in the Matlab environment and handled in Simulink interface which is adaptable to the description of indoor air balance equations. A straw bale wall incorporated in a bi-climatic chamber will serve as experimental set-up for a preliminary validation. Two simple case studies that highlight the moisture buffer effect are considered: a heat shock and a hydric shock on one side of the wall. Adding heat or moisture to a room are the two mechanisms that allow modifying its relative humidity. These distinct tests and the concomitant modeling should pave the way to a deeper understanding of the specificity of CBM materials, in particular to assess the impact of moisture buffering and latent heat phenomena on energy consumption and internal conditions during the trial. Although many publications describe the development of similar models, it is the confrontation with experimental data involving CBM that is central here.

\section{Experimental tests}

The tested straw bale panel assembly was produced by the Belgian company Paille-Tech. Straw bales are used as infill in a wood frame made of $45 \times 175 \mathrm{~mm}$ lumbers. Vertical studs are spaced at $345 \mathrm{~mm}$ on centre with bales stacked vertically between them. The resulting preferential orientation of straws is parallel to wall depth. To complete the structure, the frame was braced on the external side with breathable wood particle boards. On the internal side, the straw bales were covered with a $40 \mathrm{~mm}$ earth plaster.

The climatic chamber was made modular with a fixed base and some movable parts. A diagram illustrating the operation of a test wall is shown in Figure 1. The position of the floor and front chamber panels can be adjusted to fit the dimensions of the hosted wall. Each structural panel composing the chamber was made from $100 \times 38 \mathrm{~mm}$ timbers wood-frame closed with $18 \mathrm{~mm}$ OSB bracing. Extruded polystyrene provides thermal insulation and the inside surface were covered with a moisture barrier layer to avoid moisture penetration from the inside air volume. The chamber panels were firmly fixed around the test wall with multiple bolts and compressible air tight joints on contact area. 

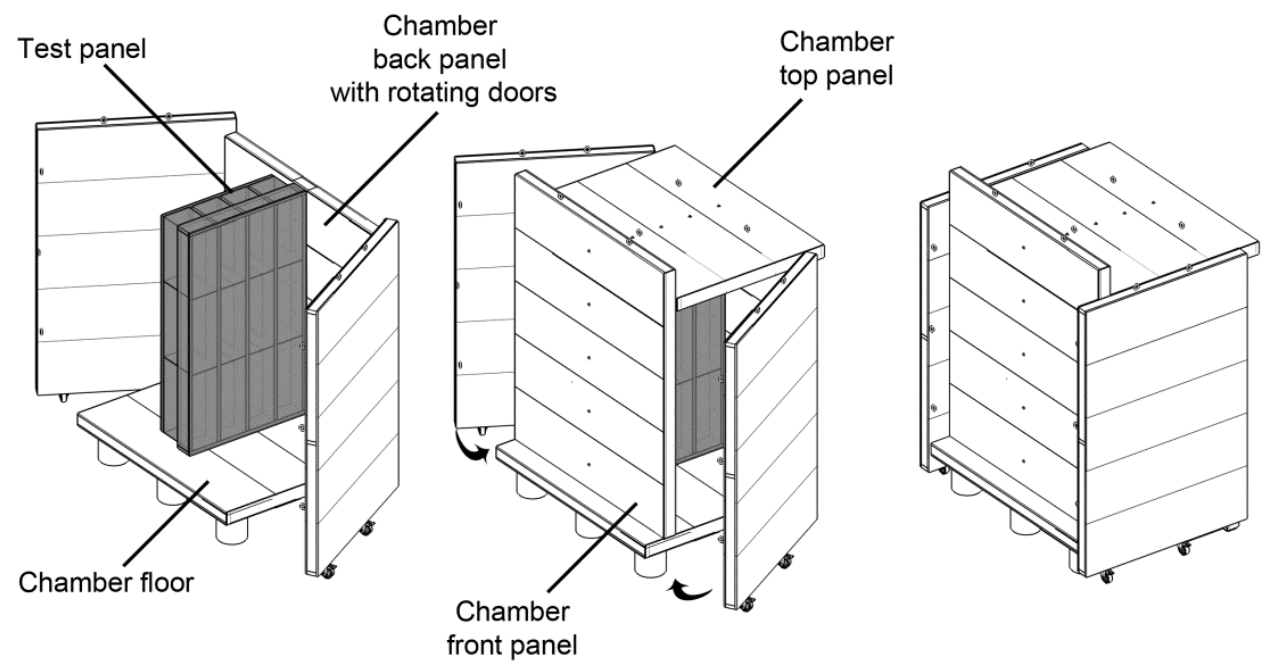

Figure 1. Modular climate chamber design

Once the straw wall was anchored in the climate chamber, two air zones ensued on either of its sides (Fig. 2). The first air volume (zone-1), on the side of plaster, is $2.84 \mathrm{~m}^{3}$ and was provided with:

- A $2 \mathrm{~kW}$ pulsed air heater that is continuously monitored by a wattmeter;

- a water vapor generation device consisting of a water bucket and a ventilator oriented towards water surface. The bucket lies on a scale for water mass monitoring.

The hydric and heat shock case studies took place in that zone, respectively through injection of water vapor or heat in the air volume. The second air volume (zone-2), on the side of the particle board, is $4.01 \mathrm{~m}^{3}$ and its climate was not controlled during the test.

Six temperature/Relative-Humidity sensors were used to provide the data intended to be compared to simulations. Those sensors were dedicated to the measurement of hygrothermal conditions in all interior and exterior air domains. The first two sensors were used to measure the hygrothermal conditions in zone-1, two other were placed in zone- 2 and the last two for measuring the external environment, i.e. the climate in the hangar that houses the climate chamber (Fig. 2).

\subsection{Case Study 1: Heat shock set-up}

At the beginning of the test, the control circuit of the heater was activated. The latter is composed of a PID controller adjusted to maintain the temperature at $35^{\circ} \mathrm{C}$ during six days. After this period, the heating system was switched off but the hygrothermal monitoring system was maintained for a further six days while the room naturally returned to equilibrium. The cumulated energy injected in zone-1, which was calculated from the readings of the heater wattmeter, is shown on Figure 3a for the entire test period. The monitored instantaneous power shows a steep initial rise to counter the chamber inertia and stabilizes around $120 \mathrm{~W}$ after one day of experiment. As soon as the heating circuit was turned off, the output of the wattmeter drops around $20 \mathrm{~W}$ which corresponds to the power needed for running the heater ventilator. The latter was kept active in order to ensure a good air circulation in zone-1.

The RH in the room is expected to decrease through the modification of the vapor saturation pressure in the air. The straw bale wall should reduce the effect observed in a room where no moisture exchange can take place. 


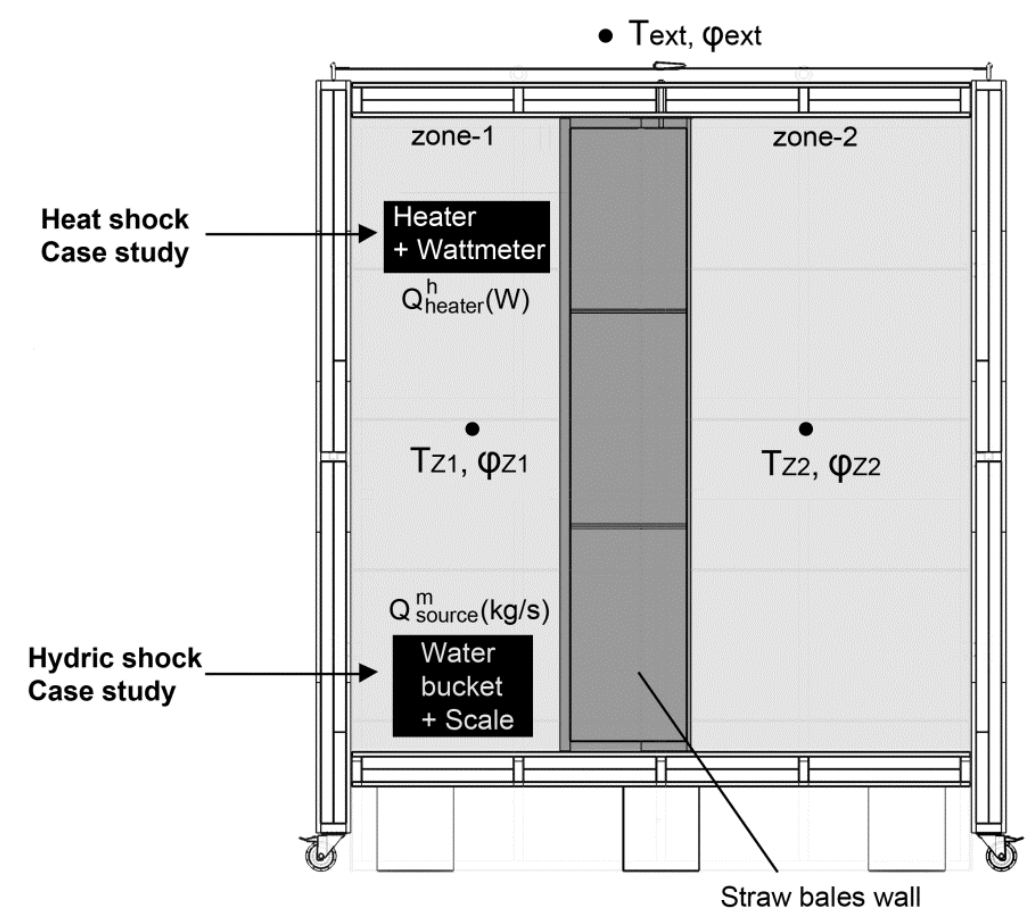

Figure 2. Climate chamber, hygrothermal sensors and case studies set-ups

\subsection{Case study 2: Hydric shock set-up}

This second test consists in adding water vapor in zone-1 with the vapor generation device. The water bucket was filled before the test and then placed on the scale. Once both doors of the chamber were closed, the ventilator was activated for 12 days causing water to be added to the indoor air at a relatively constant rate. Figure $\mathbf{3 b}$ shows the cumulated moisture injected in zone-1 during the second case study. The RH in the room is expected to increase but the straw bale wall should fight against this modification through moisture exchange. In other terms, the RH rise in a room without any hygroscopic materials is supposedly higher.

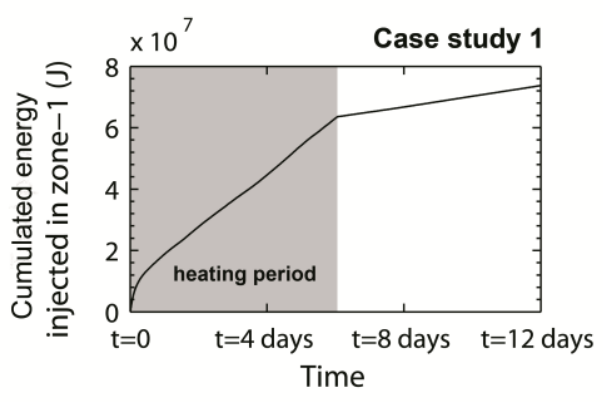

(a)

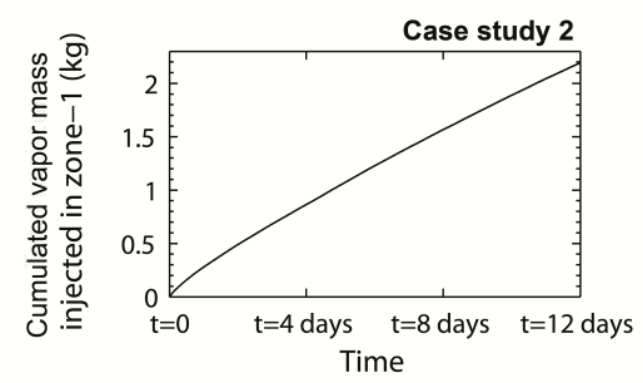

(b)

Figure 3. (a) Cumulated energy injected in zone-1 during the first case study and (b) cumulated vapor mass injected in zone-1 during the second case study

\section{Hygrothermal model}

In the modeling exercises, the unknowns are the temperature $T$ and absolute humidity $\omega$ in each air zone of the bi-climatic chamber. The absolute humidity can also be expressed in terms of partial vapor pressure. These quantities must be determined from balance equations performed on each zone, while considering hygrothermal interaction with the straw bale wall and the panels of the chamber, loads from air infiltration, and for zone-1, the internal heat or moisture source depending on the case study. The model is divided in two distinct sub-parts which are coupled to obtain the overall response of 
interior air domain.

\subsection{BEHAM sub-part}

The first model sub-part is dedicated to the description of the coupled heat and moisture transfers in the envelope, i.e. the straw bale wall and the constitutive panels of the chamber. It was developed using the COMSOL Multiphysics software directly transposed into Matlab language. This general computational tool provides a powerful solution for solving partial differential equations by the finite element method. Furthermore, its flexibility allows modifying at will the physical phenomena considered in the different envelope elements.

\section{Balance equations}

Two PDE balance equations are solved simultaneously in one dimension:

$$
\begin{gathered}
\rho_{0} \frac{\partial u}{\partial \varphi} \frac{\partial \varphi}{\partial t}=-\frac{\partial}{\partial x}[-\underbrace{\frac{\delta_{a} \frac{\partial p_{s a t}}{\partial T} \frac{\partial T}{\partial x}-\frac{\delta_{a} p_{\text {sat }}}{\mu} \frac{\partial \varphi}{\partial x}}{\text { Heat balance (PDE) }}}_{\begin{array}{c}
\text { Humidity balance (PDE) } \\
\boldsymbol{j}_{x}^{M_{v}}
\end{array}}] \\
\rho_{0}\left(c_{0}+u c_{l}\right) \frac{\partial T}{\partial t}+\rho_{0} \frac{\partial u}{\partial \varphi} \frac{\partial \varphi}{\partial t} c_{l}\left(T-T_{0}\right)=\frac{\partial}{\partial x}\left[\lambda_{\text {eff }} \frac{\partial T}{\partial x}\right]-\frac{\partial \boldsymbol{j}_{x}^{\boldsymbol{M}_{v}}}{\partial x} L
\end{gathered}
$$

with $\boldsymbol{j}_{\boldsymbol{x}}^{\boldsymbol{M}_{\boldsymbol{v}}}\left(\mathrm{kg} \cdot \mathrm{m}^{-2} \cdot \mathrm{s}^{-1}\right)=$ the vapor flux density in the material. Two versions of the BEHAM model were prepared to assess how different phenomena taking place in the straw bales impacts the predicted climate in zone-1. In other terms, using various versions of the BEHAM tool, with increasing simplification level, allows evaluating which assumptions can be reasonably undertaken in the description of envelope materials behavior. The first version (BEHAM-HM) solves the two coupled PDEs, i.e. equations (1) and (2), with the Oswin's function (Oswin, 1946) for moisture storage:

$$
u(\varphi)=C *\left(\frac{\varphi}{1-\varphi}\right)^{n}
$$

where $C$ and $n$ are constant parameters. The second version (referred to as $\boldsymbol{B E H A M - H}$ ) does not take into account the moisture transfer into the wall straw and incorporates only the heat PDE; this last version corresponds to a scenario where the straw bales wall is not permeable to water transfer. The different scenarii used for the description of hygrothermal transfers in the straw bales wall are a perfect illustration of the flexibility of the software, which are highly desirable in research perspective.

In contrast to the straw bales wall where different versions of moisture storage equations are considered, only the heat transfer will be taken into account in the structural panels of the climatic chamber (justified by the presence of vapor barrier). The properties of various materials are listed in Table 1. Because this is a preliminary study, the transport coefficients are considered constant during the test. Note that the fraction of wood is taken into account in the values of thermal conductivity for the straw and polystyrene.

Table 1. Hygrothermal properties of materials

\begin{tabular}{lcccccc}
\hline & \multicolumn{7}{c}{ Table 1. Hygrothermal properties of materials } & $\boldsymbol{\mu}$ & $\boldsymbol{C}$ & $\boldsymbol{n}$ \\
Materials & $\boldsymbol{\rho}_{\mathbf{0}}$ & $\boldsymbol{\boldsymbol { c } _ { \mathbf { 0 } }}$ & $\boldsymbol{\mu}$ & $(-)$ \\
\hline XPS + wood fraction & 30 & 0.048 & 1500 & - & - & - \\
OSB & 620 & 0.13 & 1500 & - & - & - \\
Straw + wood fraction & 100 & 0.07 & 2420 & 1.5 & 0.08 & 0.42 \\
Clay plaster & 2051 & 1 & 750 & 18.5 & 0.0029 & 0.445 \\
P. board & 570 & 0.09 & 2100 & 22.5 & 0.13 & 0.29 \\
\hline
\end{tabular}

\subsection{Zone balance sub-part}

The second module of the room-scale model performs the heat and moisture balances on the air 
volumes of zone-1 and zone-2. This sub-model is built on the assumption that indoor air is perfectly mixed, implying that the unknown variables do not depend on spatial coordinates inside the air volumes. Therefore, indoor air balance equations are accounted for by ordinary differential equations (ODE).

\subsubsection{Humidity balance (ODE)}

The following loads are involved in the moisture balance in each zone: $\dot{Q}_{e, s t}^{m}\left(k g \cdot s^{-1}\right)$, the moisture exchange with the straw bales wall and $\dot{Q}_{v}^{m}\left(\mathrm{~kg} \cdot \mathrm{s}^{-1}\right)$, the moisture exchange with exterior domain by air infiltration in the zone and $\dot{Q}_{\text {source }}^{m}$ the moisture source in the zone. The humidity ODE for a zone is written:

$$
\rho_{a} V \frac{d \omega}{d t}=\underbrace{\dot{Q}_{e, s t}^{m}}_{\text {straw bales wall }}+\underbrace{\dot{Q}_{v}^{m}}_{\text {air infiltration }}+\underbrace{\dot{Q}_{\text {source }}^{m}}_{\text {moisture source }}
$$

where

$$
\begin{gathered}
\dot{Q}_{e, s t}^{m}=A_{\text {st }} \frac{\left(p_{v_{\text {surf }}}^{\text {st }}-p_{v}\right)}{Z_{\text {surf }, \text { int }}} \\
\dot{Q}_{v}^{m}=\dot{m}\left(\omega_{\text {ext }}-\omega\right)
\end{gathered}
$$

with $A_{s t}\left(m^{2}\right)=$ the exchange area of the straw bales wall, $\dot{m}\left(\mathrm{~kg} \cdot \mathrm{s}^{-1}\right)=$ the air infiltration rate in the zone, $p_{v}^{\text {surf }}=$ the vapor partial pressure at straw bale wall surface and $p_{v}=$ the vapor partial pressure in the zone. The infiltration rates in zone-1 and zone- 2 were not measured in this study and their values were estimated roughly in preliminary simulations. The parameter was assigned with $\dot{m}=$ $0.0005 \mathrm{~kg} \cdot \mathrm{s}^{-1}$ for both zones.

\subsubsection{Heat balance (ODE)}

The following loads are involved in the heat balance in each zone: $\dot{Q}_{e, s t}^{h}(W)$, the heat exchange with the straw bales wall, $\dot{Q}_{e, c h}^{h}(W)$ the heat exchange with the chamber panels, $\dot{Q}_{v}^{h}(W)$, the heat exchange with exterior domain by air infiltration in the zone, $\dot{Q}_{\text {source }}^{h}(W)$ the heat load from the heating system and the ventilators. The heat ODE for a zone is written:

$$
\rho_{a} V\left(c_{a}+\omega c_{v}\right) \frac{d T}{d t}=\underbrace{\dot{Q}_{e, s t}^{h}}_{\text {straw bale wall }}+\underbrace{\dot{Q}_{e, c h}^{h}}_{\text {chamber panels }}+\underbrace{\dot{Q}_{v}^{h}}_{\text {air infiltration }}+\underbrace{\dot{Q}_{\text {source }}^{h}}_{\text {Heat source }}
$$

where

$$
\begin{gathered}
\dot{Q}_{e, s t}^{h}=A_{s t} \frac{\left(T_{\text {surf }}^{\text {st }}-T\right)}{R_{\text {surf }, \text { int }}} \\
\dot{Q}_{e, c h}^{h}=A_{\text {chtot }, Z i} \frac{\left(T_{\text {surf }}^{\text {ch }}-T\right)}{R_{\text {surf }, \text { int }}} \\
\dot{Q}_{v}^{h}=\dot{m} c_{a}\left(T_{\text {ext }}-T\right)
\end{gathered}
$$

where $A_{\text {chtot }, Z i}\left(\mathrm{~m}^{2}\right)=$ the total surface of chamber panels in contact with zone- $i$. The heater load is obtained directly by the power measurement circuit linked to the heater. It is important to observe that all the chamber panels in contact with a zone are accounted for together, as a single load.

\subsection{Sub-parts integration}

The two subparts of the room-scale hygrothermal model were combined within Simulink ${ }^{l}$ modeling tool, which is included in Matlab GCT. Simulink is a block diagram environment dedicated to the resolution of dynamical systems and fully integrated with Matlab standard tools and functions. Combining ODE and PDE based models requires the use of a particular block called an S-Function

\footnotetext{
${ }^{1}$ http://www.mathworks.com/products/simulink/
} 
(system-function). An S-Function is nothing more than a customizable code that describes the behavior of a dynamical system and allows the user to create new blocks in order to enhance Simulink capabilities. Designing an S-Function means defining the state(s) of the system, the derivative function of the continuous state(s), the update function of the discrete state(s), and the relationships that link the outputs to the inputs and states of the system. Solving the room balance-equations with a PDE model for envelope elements requires the use of such custom S-Function associated with standard blocks available in the default Simulink library.

Three S-Functions were created here: one for describing the transient behavior of the straw bales wall, another for describing the transient behavior of the chamber panels in contact with zone-1, and a last S-Function for the chamber panels in contact with zone-2. The outputs of those S-Functions are the surface hygrothermal conditions on interior side, which make the bridge between the BEHAM and the zone balance equations. Their inputs are the hygrothermal conditions in exterior and interior air volumes, needed to define the boundary conditions on all chamber envelope elements. Each of the Sfunctions is characterized by hybrid states: The hygrothermal conditions inside envelope materials are updated at a regular time interval, using conditions in air zones and the external climate for the computation of boundary conditions; wall surface conditions are thus updated on a discrete time basis, with a regular major time step $(\sim 15 \mathrm{~min})$. Between those major time intervals, the wall surface conditions are maintained constant and still intervene in room balance equations. The latter are based on a continuous states description. The two zone balance ODEs (Equations (4) and (7)) are solved with standard blocks included in the library of Simulink and follow a minor time stepping $(\sim 1 s)$.

\section{Results and discussions}

\subsection{Case study 1: Heat shock}

Figure 4 shows the experimental evolution of temperature and vapor partial pressure in zone-1 as well as the room-scale model outputs, for the two versions of the BEHAM sub-part used in the room-scale model to characterize the straw bale wall. Regarding indoor humidity conditions, we observe that the version of the hygrothermal model that considers moisture transfer in the straw bale wall (BEHAM$H M$ ) provides a curve appearance consistent with the experimental measurement. The model with the BEHAM-H module results in a poor characterization of indoor humidity; the vapor pressure in zone-1 follows exterior solicitations due to air infiltration, which is the only humidity load accounted for. The distinction between BEHAM versions is more difficult to observe for indoor temperature simulation. It can be seen that accounting for moisture transport in the straw bales wall translates into a better fit of experimental data. The BEHAM-H model predicts a room temperature higher than experimentally observed. This can be explained by the latent heat effect. When the temperature rises in zone-1, the water saturation pressure rises consequently and the RH of the room has a tendency to decrease. Due to the moisture buffer effect, the plaster reacts immediately by releasing some vapor towards the indoor air volume. The water molecules that desorb from the plaster inner matrix require some heat to perform this phase change. In consequence, the hygroscopic wall act indirectly also as a temperature buffer. The BEHAM module which does not account for moisture transfer in the straw bales wall will naturally result in a higher temperature prediction in zone-1. 

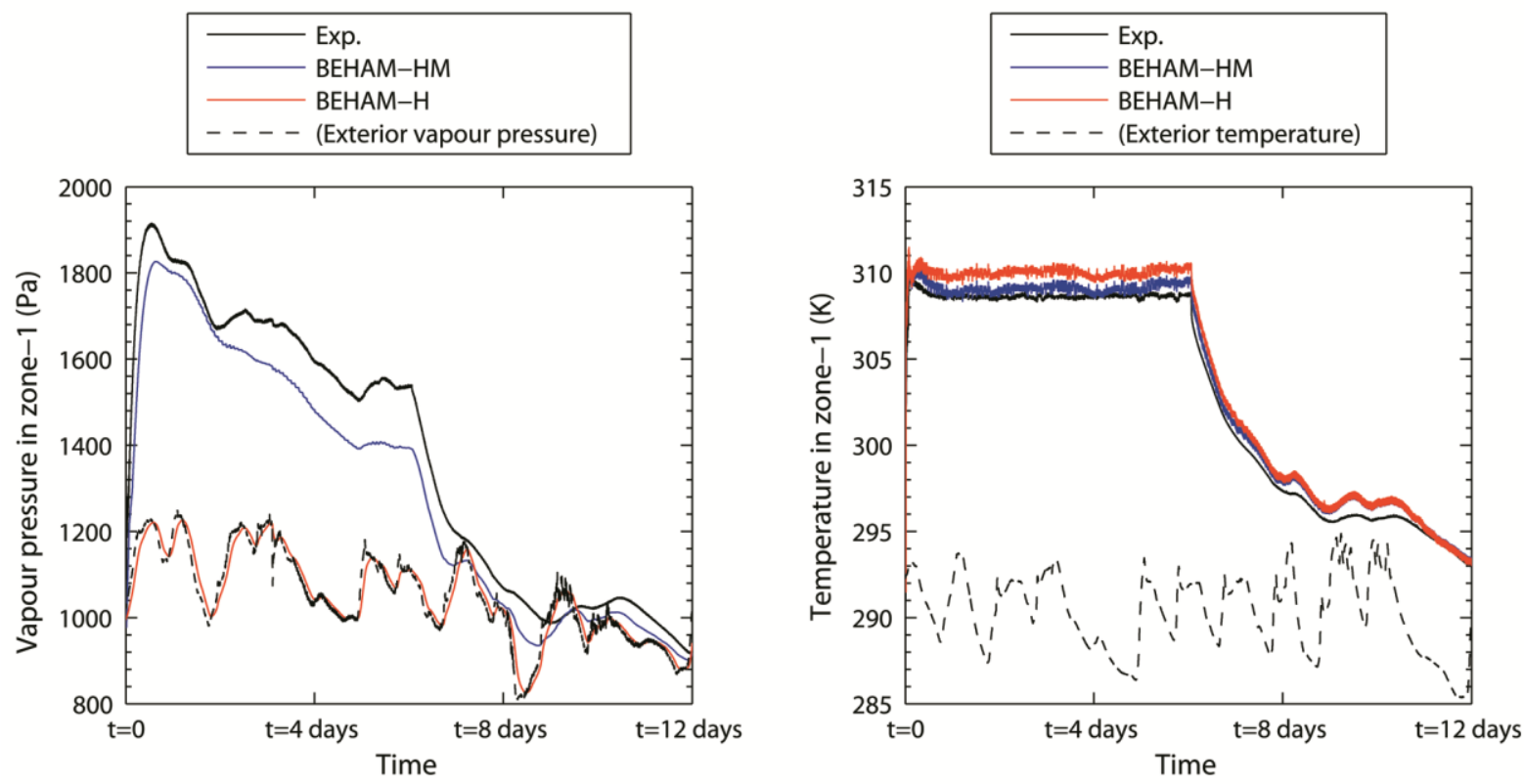

Figure 4. Model outputs compared to experimental results; vapor pressure and temperature in zone-1 (heat shock)

In order to illustrate more clearly the moisture buffer effect, Figure 5 shows the RH evolution in zone1 according to the models versions compared to experimental data. It can be observed that without the moisture exchange in the straw bale wall (a scenario corresponding to BEHAM-H), the heat shock will cause a drop of $\mathrm{RH}$ in zone-1 followed by a stabilization around $20 \% \mathrm{RH}$ during the entire heating period. In contrast, the measurement data shows that the initial RH drop is rapidly compensated by a moisture release from the wall which causes the RH to rise again. At the beginning of the test, the measured $\mathrm{RH}$ in the room was $48 \% \mathrm{RH}$. After the initial drop that brings the $\mathrm{RH}$ to $22 \%$, the straw bales wall reaction is observable in the form of a slow rise of RH which reaches $34 \%$ in approximately 10 hours. This response of the breathable wall is thus directly noticeable by analysis of the indoor RH. The BEHAM-HM demonstrates a good fit to experimental data.

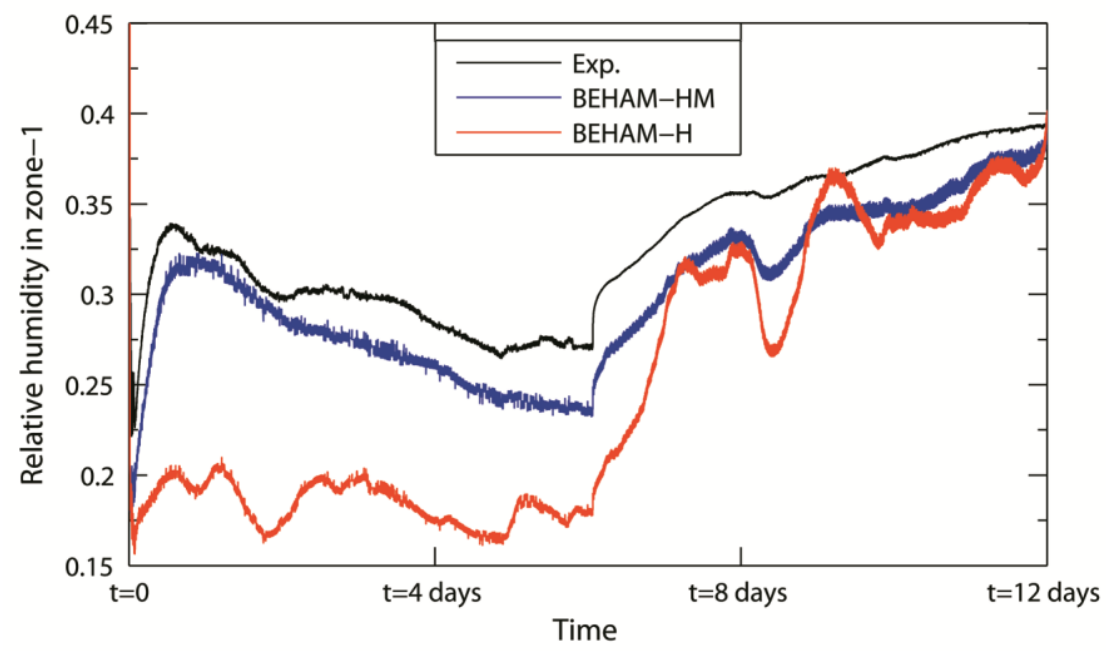

Figure 5. Model versions outputs compared to experimental results; RH in zone-1 (heat shock) 
The modeling results for the second case study are shown on Figure 6. The simulated vapor pressure and temperature in zone-1 are compared to the sensors readings for the two versions of the COMSOL model, similarly to the precedent case study. Several observations can be made. When the BEHAM-H module is used, the vapor pressure in the room is predicted to reach very quickly its saturation value.
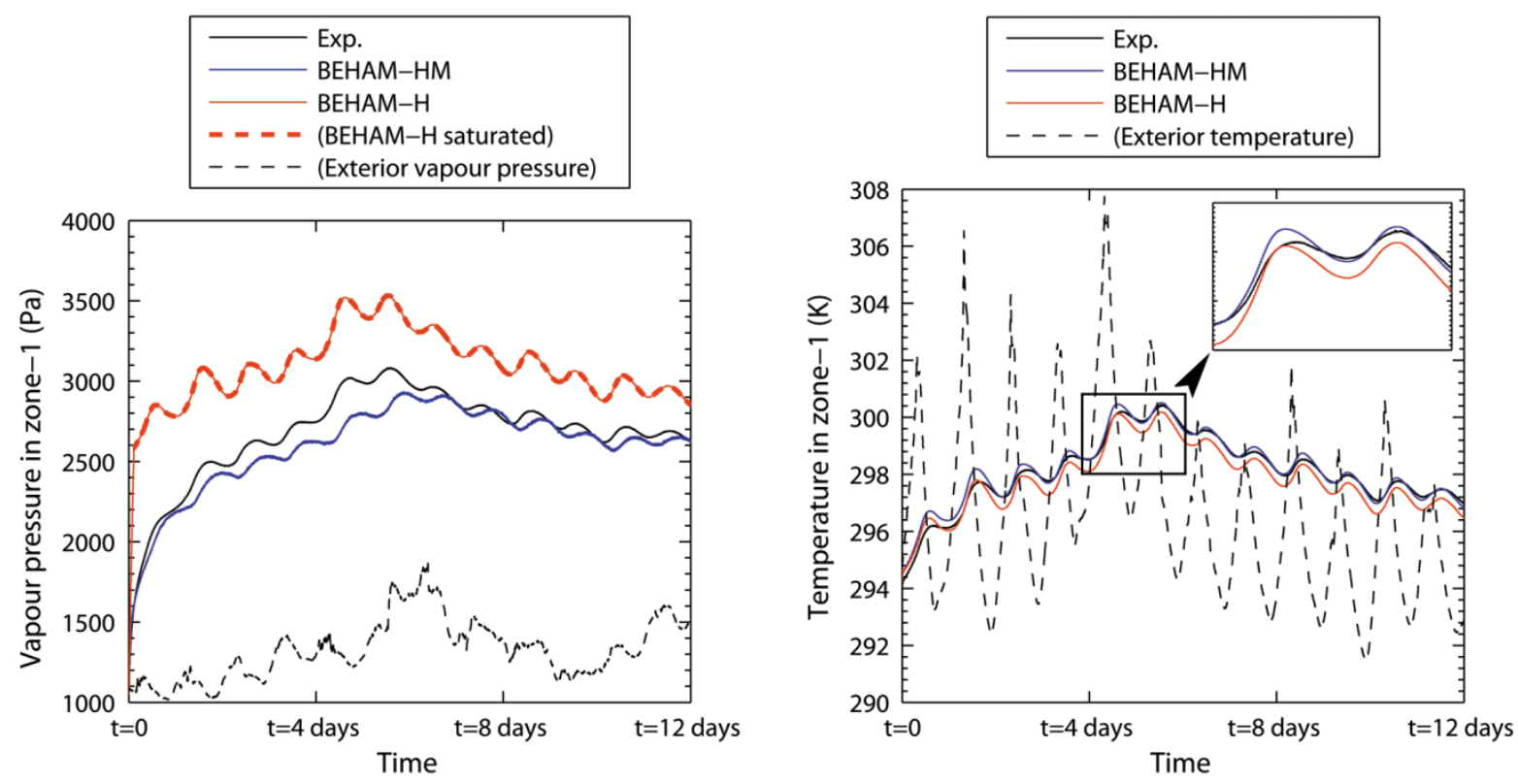

Figure 6. Model outputs compared to experimental results; vapor pressure and temperature in zone-1 (hydric shock)

Indeed, under a impermeable straw bale wall assumption, the air infiltration in the room is not sufficient to desaturate the air given the constant supply of water through the moisture generation device. Moreover, the BEHAM-H model output exhibits an air temperature that is lower than observed for the entire test period. This could be explained by some latent heat effect: as the wall constantly absorbs water from zone-1, heat is release when water molecules adsorbs in the porous materials of the wall.

In contrast, the BEHAM-HM model results in a good prediction of indoor variables evolution. The temperature is higher than observed for the two first days but the difference is strongly reduced afterwards. The biggest issue is the phase shift noted between the experimental and the modeled vapor pressure. The sensors reveal that the small fluctuations of vapor pressure in the room are synchronised with temperature variations whereas it is delayed in the simulation. This difference could be explained by the fact that in such a preliminary study we used many strong assumptions: constant material parameters, estimated surface coefficients, no hysteresis effect nor liquid transport.

\subsection{Example of wall performance analysis}

The great advantage of the hygrothermal model at zone-scale lies in fact that it allows analyzing the real heat and mass flows occurring at the various interior surfaces of the envelope. By real, we mean that the mutual exchange between envelope and indoor air are accounted for and not only the transfers from indoor air towards the envelope (as it is the case when BEHAM models are used alone). This is illustrated here for the first case study (heat shock). For example, one can estimate the impact of different moisture transfer mechanisms included in the BEHAM model on the heat flow exchange between the heated indoor environment and the straw bales wall, in order to identify potential benefits for the occupant in terms of comfort of energy consumption. In particular, many interrogations persist in the evaluation of latent heat effect on building energy performance (BRE, 2002). During a $35^{\circ} \mathrm{C}$ thermal shock, energy performance benefits would lie principally in the reduction of cooling loads and comfort benefits in keeping the Predicted Percentage of Dissatisfied (PPD) value as low as possible as 
defined in ISO 7730. The PPD value will determine how many occupants will fall outside the limits of comfort by feeling to cold or to warm. This indicator depends on the metabolic level of occupants as well as on their clothing. However, the full analysis of PPD values is beyond the scope of this chapter. Some simple criteria were chosen as indoor comfort and energy performance indicators to characterize zone-1 under the heat shock scenario. Those indicators are the zone temperature, the zone relative humidity, the surface temperature of the straw bales wall and the thermal flux from zone air towards the straw bales wall. The criteria were computed for both versions of the envelope model used for the study of the straw bales wall. Results are presented in Table 2 for the heating period (see Fig. 3a).

It can be observed that the moisture transfers happening in the straw bales wall indeed plays a significant role in indoor climate regulation. During the heating period, both indoor air and surface temperature are lower when hygroscopic effects are considered. In addition, the RH level is maintained closer to the initial room value while it drops more severely if the straw-bales wall is considered as impermeable to water transfers. Under BEHAM-HM assumptions, the heat flux going from the room to the straw bales wall is increased by $17.3 \%$ in comparison to the no-moisture-transfer model (BEHAM-H). This result is particularly significant in reducing cooling load and improving energy storage during an overheated period. When analyzing the results presented in Table 2, it should be remembered that only one wall is able to exchange moisture in zone-1; the straw bale wall only represents an area of $3.66 \mathrm{~m}^{2}$ on the total interior surface of $10.43 \mathrm{~m}^{2}$ in zone-1. The benefits of hygroscopic walls on all interior surfaces could be really noticeable, this time also in terms of indoor temperature.

Table 2. Averaged indoor comfort criteria during heating period of case study 1

\begin{tabular}{cll}
\hline & BEHAM-HM & BEHAM-H \\
\hline $\boldsymbol{T}_{\boldsymbol{Z} \mathbf{1}}$ & 309.0185 & 309.9622 \\
$\boldsymbol{\varphi}_{\boldsymbol{Z} \mathbf{1}}$ & 0.2780 & 0.1849 \\
$\boldsymbol{T}_{\text {surf } \boldsymbol{Z} \mathbf{Z 1}}^{\boldsymbol{t}}$ & 307.2926 & 308.4902 \\
$\left(\dot{\boldsymbol{Q}}_{\boldsymbol{e}, \boldsymbol{s t}}^{\boldsymbol{H}}\right)_{\mathbf{Z 1}}$ & -31.6167 & -26.9628 \\
\hline
\end{tabular}

\section{Conclusion}

A hygrothermal model at room-scale was developed by combining a BEHAM research model to zone balance equations into the Simulink computational environment. The objective was to show how the impact of CBM on indoor climate can be characterized. A preliminary confrontation of this model with experimental data from a heat shock and a hydric shock on a straw bale panel allows to:

- Highlight the benefits of a hygrothermal model at room-scale which consider the coupled and transient response of hygroscopic envelopes, particularly with regard to the characterization of the actual flows from the envelope to the indoor air zone;

- confirm the capabilities of hygroscopic materials to improve the comfort of the building;

- quantify this effect during one of the proposed scenarii;

- address the current weaknesses of the simulation, namely the lack of precise knowledge of the convective transfer coefficients at envelope surfaces, the rate of ventilation in zones and all the materials properties. Because the estimation of those parameters is difficult to measure experimentally, it is worth noticing that Matlab provides the ability to easily use inverse modeling modules that can facilitate the process.

The experimental tests used here for the purpose of model validation are really simple. In real environments, the ventilation rates have a major role on indoor climate whereas they were kept really low during the presented tests. However, we showed that hygrothermal models are getting more and more powerful and seem ready for the confrontation to a large range of experimental case studies, eventually with realistic solicitations. Future work should implement the validation tests which illustrate various operating conditions of the building in the perspective of highlighting and quantifying other qualities of hygroscopic structures and crop-based materials. 


\section{References}

BRE. (2002). Final Report on the construction of the Hemp Houses at Haverhill, Suffolk. UK: Building Research Establishment. Retrieved from http://projects.bre.co.uk/hemphomes/HempHousesatHaverhillfinal.pdf

Hens, H. S. L. C. (2005). Annex 41 - Whole building heat, air and moisture response. in Proc. 26th AIVC Conference. Brussels, Belgium: AIVC.

Janssens, A., Woloszyn, M., Rode, C., Sasic-Kalagasidis, A., \& De Paepe, M. (2008). From EMPD to CFDoverview of different approaches for Heat Air and Moisture modeling in IEA Annex 41. In Proc. IEA ECBCS Annex 41 Closing Seminar. Copenhagen, Denmark: IEA.

Oswin, C. (1946). The kinetics of package life. III. The isotherm. Journal of the Society of Chemical Industry, 65(12), 419-421.

Rode, C., \& Grau, K. (2008). Moisture Buffering and its Consequence in Whole Building Hygrothermal Modeling. Journal of Building Physics, 31(4), 333-360. doi: 10.1177/1744259108088960

Rode, C., \& Woloszyn, M. (2007). Whole-Building Hygrothermal Modeling in IEA Annex 41. in Proc. 10th Intl. Conf. Thermal Performance of Exterior Envelopes of Whole Buildings, Clearwater, USA: ORNL.

Salonvaara, M., Ojanen, T., Holm, A., Künzel, H. M., \& Karagiozis, A. N. (2004). Moisture buffering effects on indoor air quality-experimental and simulation results. in Proc. 9th Intl. Conf. Thermal Performance of Exterior Envelopes of Whole Buildings, Clearwater, USA: ORNL.

Sasic Kalagasidis, A. (2004). HAM-Tools - An Integrated Simulation Tool for Heat, Air and Moisture Transfer Analyses in Building Physics: Chalmers University of Technology.

Tariku, F., Kumaran, K., \& Fazio, P. (2010). Integrated analysis of whole building heat, air and moisture transfer. International Journal of Heat and Mass Transfer, 53(15-16), 3111-3120. doi: 10.1016/j.ijheatmasstransfer.2010.03.016

Tran Le, A., Maalouf, C., Mai, T., Wurtz, E., \& Collet, F. (2010). Transient hygrothermal behaviour of a hemp concrete building envelope. Energy and Buildings, 42(10), 1797-1806.

van Schijndel, A. W. M. (2009). Integrated modeling of dynamic heat, air and moisture processes in buildings and systems using SimuLink and COMSOL. Building Simulation, 2(2), 143-155. doi: 10.1007/s12273009-9411-x

Woloszyn, M., \& Rode, C. (2008). Tools for performance simulation of heat, air and moisture conditions of whole buildings. Building Simulation, 1(1), 5-24. doi: 10.1007/s12273-008-8106-z 
Nomenclature

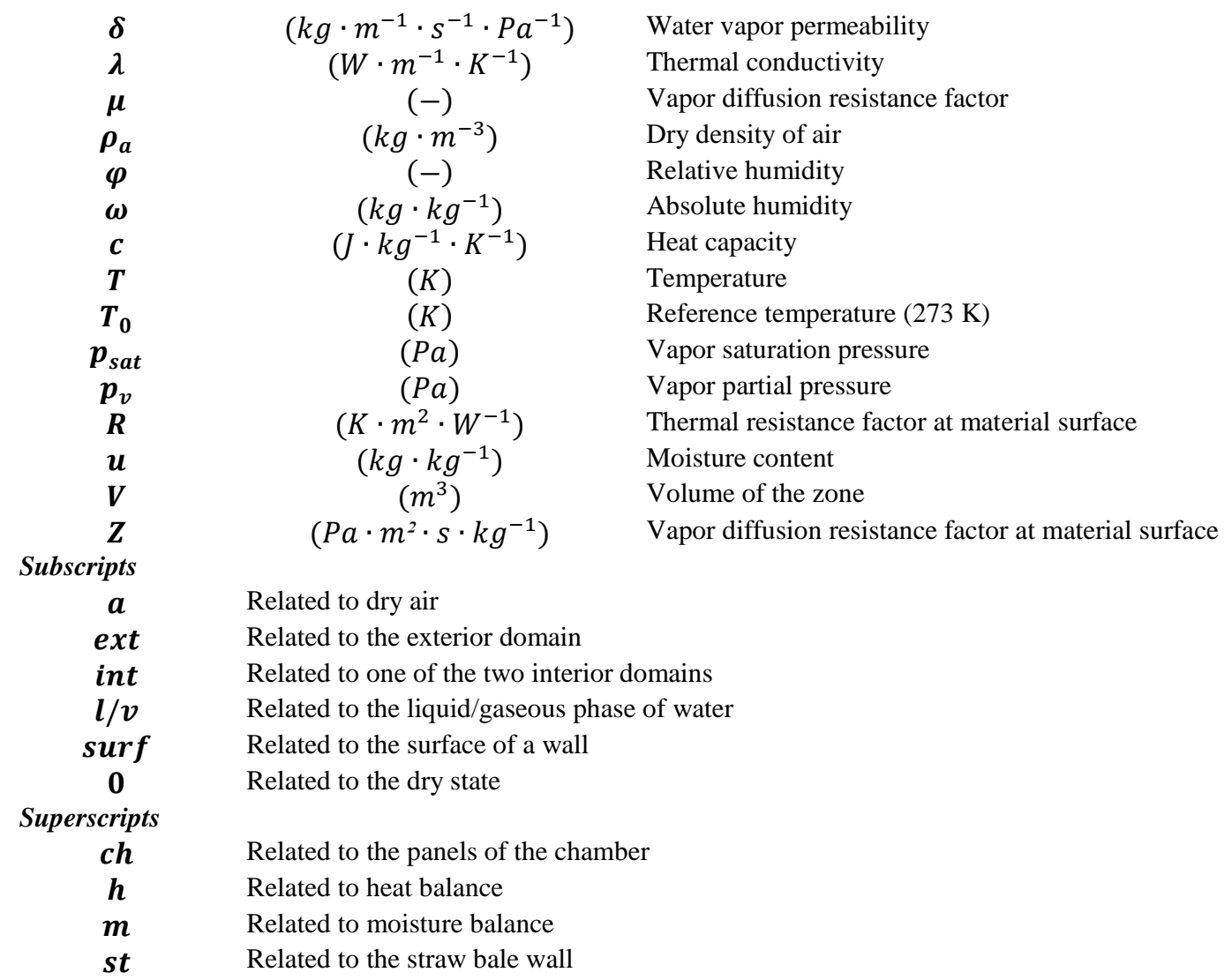

